\title{
The Effect of Oxygen Potential on the Surface Self-Diffusion Coefficient of Silver*
}

\author{
By Kazuhiro Yoshihara** and Kazuyoshi Nii**
}

\begin{abstract}
The surface self-diffusion coefficient of silver was measured by the grain boundary grooving method in atmospheres with various oxygen potentials. In an argon atmosphere with $\boldsymbol{P}_{\mathbf{O}_{2}}=\mathbf{1 0}^{-11} \mathrm{~Pa}$, the surface self-diffusion coefficient can be expressed by the following equation:

$$
D_{s}=0.249 \exp (-166 \mathrm{~kJ} / \mathrm{mol} \cdot R T) \mathrm{m}^{2} / \mathrm{s}(973-1073 \mathrm{~K}) \text {. }
$$

This equation coincides well with Gjostein's experimental formula for $f c c$ metals.

As the oxygen potential increased from $P_{\mathrm{O}_{2}}=10^{-11} \mathrm{~Pa}$, the surface self-diffusion coefficient decreased and became minimum at $P_{\mathrm{O}_{2}}=10^{-2} \mathrm{~Pa}$. At $P_{\mathrm{O}_{2}}=10^{-2} \mathrm{~Pa}$, the temperature dependence of the surface self-diffusion coefficient cannot be expressed by Arrhenius' equation. As the oxygen potential further increased above $P_{\mathbf{O}_{2}}=10^{-2} \mathrm{~Pa}$, the surface self-diffusion coefficient increased and the activation energy for the diffusion at $P_{\mathbf{O}_{2}}=10^{5} \mathrm{~Pa}$ was smaller than that at $P_{\mathrm{O}_{2}}=10^{-11} \mathrm{~Pa}$. The surface self-diffusion coefficient at $P_{\mathrm{O}_{2}}=10^{5} \mathrm{~Pa}$ can be expressed by the following equation:

$$
D_{s}=1.71 \times 10^{-3} \exp (-106 \mathrm{~kJ} / \mathrm{mol} \cdot R T) \mathrm{m}^{2} / \mathrm{s}(973-1073 \mathrm{~K}) \text {. }
$$

The following model for the surface structure of silver at various oxygen potentials has been proposed. At $\boldsymbol{P}_{\mathbf{O}_{2}}=10^{-11} \mathrm{~Pa}$, oxygen ions are hardly adsorbed and the surface of silver remains clean. At $P_{\mathbf{O}_{2}}=10^{-2} \mathrm{~Pa}$, oxygen ions are adsorbed locally, and neighboring silver atoms are ionized and stabilized. Then the number of sites, from which mobile ad-atoms that contribute to the diffusivity are produced, decreases. At $P_{\mathbf{O}_{2}}=10^{5} \mathrm{~Pa}$, the adsorbed oxygen ions are arranged in order and a 2-dimensional ionic crystal is formed on the surface. Then the jump distance becomes longer and the activation energy for the diffusion becomes smaller by an overlapping of long range Coulomb's force. As a result, the surface self-diffusion coefficient increases.
\end{abstract}

(Received May 7, 1979)

\section{Introduction}

Surfaces of solid metals play an important role in such phenomena as sintering, gas permeation, gas adsorption, friction and joining. An understanding of the mechanism of these processes, therefore, requires the information on the metal surfaces. Surfaces are easily affected by the adsorption of foreign atoms, which reflects directly on the migration of atoms on surfaces. Therefore, by observing the changes of surface self-diffusion coefficients with atmospheres, it is possible to deduce the changes of surface states, namely, the changes of surface structures with adsorption ${ }^{(1)}$.

Surface self-diffusion coefficients of metals either increase or decrease with atmospheres.

* This paper was originally published in Japanese in J. Japan Inst. Metals, 42 (1978), 492.

** National Research Institute for Metals, Meguroku, Tokyo 153, Japan.
As for the effects of atmospheres on surface self-diffusion coefficients, the following mechanisms have been proposed. The mechanisms for increasing of surface self-diffusion coefficients are as follows: (1) Skating mechanism; a metal atom can move a long distance in an adsorbed layer as if it were skating ${ }^{(2)}$. (2) Two dimensional liquid model; adsorbed atoms and metal atoms form a two dimensional liquid compound, so a metal atom can move as if it were diffusing in liquid ${ }^{(3)}$. (3) Non-localized state theory; the existence of adsorbed atoms increases the probability of forming dimers and trimers of surface metal atoms and they can move more freely than single atoms $\mathrm{do}^{(4)}$. The mechanisms for decreasing of surface self-diffusion coefficients are as follows: (1) Immobile pair model; adsorbed atoms and metal atoms form immobile pairs ${ }^{(5)}$. (2) Ledge pinning model; the motion of ledges is restricted by the interaction between adsorbed atoms and ledges ${ }^{(6)}$. (3) The hindrance of the formation of ad-atoms; when foreign atoms are

1979 Vol. 20 
adsorbed at kink sites, the formation of adatoms which contribute to the diffusivity is hindered $^{(1)}$. Each mechanism, however, has not the universality that can apply to all the systems of metals and atmospheres.

In this report, to get the systematic understanding of the effect of atmospheres on surface self-diffusion coefficients of metals, the surface self-diffusion coefficient of silver has been measured by the grain boundary grooving method in atmospheres with various oxygen potentials.

\section{Experimental Method}

\section{Sample}

Granular silver with high purity (99.99\%) was melted in a crucible in the air and washed by nitric acid, then remelted in an argon- $1 \%$ hydrogen atmosphere and slowly cooled. The silver button which had many large grains were cut into a plate, the size of which is $12 \times 12 \times 5$ $\mathrm{mm}$. In order to make the grain size appropriate and grain boundaries stable so as not to move during measurements, the plate was cold-rolled about $7 \%$, then it was well annealed at $1100 \mathrm{~K}$ in a pure argon atmosphere. The average grain size of the sample was about $5 \times 5 \mathrm{~mm}$. The measurements were carried out on the straight grain boundary with good symmetry.

After the sample was polished by a silicon carbide paper and a diamond paste, it was electrolytically polished in the bath of sodium acetate - sodium thiosulfate - acetic anhydride $^{(7)}$. Then it was washed by distilled water in an ultrasonic bath. After that, it was ion bombarded for $10 \mathrm{~min}$ in an argon atmosphere $\left(10^{-2} \mathrm{~Pa}\right)$. The acceleration voltage of the argon ion was $0.7 \mathrm{kV}$. The obtained flat surface was analyzed by AES. This showed that only the very small amount of chlorine was present on the surface as an impurity.

\section{Annealing}

Annealing was carried out in the temperature range between 973 and $1073 \mathrm{~K}$ with oxygen potentials between $10^{-11} \mathrm{~Pa}$ and $10^{5} \mathrm{~Pa}$. The sample was inserted into a furnace which had been adjusted at the given temperature and the oxygen potential. To avoid the vaporization of silver during annealing, the sample was covered with a silver plate.

In the case of $P_{\mathrm{O}_{2}}=10^{5} \mathrm{~Pa}$, the pure oxygen was used, which was passed through silica gel. In the case of $P_{\mathrm{O}_{2}}=10^{-11} \mathrm{~Pa}$, the purified commercial argon gas $\left(P_{\mathrm{O}_{2}}=10 \mathrm{~Pa}\right)$ which was passed through titanium tips heated at $1200 \mathrm{~K}$ was used. The oxygen potentials between $10^{5}$ $\mathrm{Pa}$ and $10^{-11} \mathrm{~Pa}$ were obtained by regulating the flowing ratio of these gases. The oxygen potential of an atmosphere was always monitored by a solid electrolyte cell using stabilized zirconia.

\section{The measurements of grain boundary groove widths}

After annealing, the photograph of the interferogram of grain boundary grooves was taken by an interference microscope with a sodium lamp. Ten lines in the interferogram were measured by a comparator and their average value was determined as a grain boundary groove width.

\section{The calculation of the surface self-diffusion coefficient}

If a grain boundary groove is formed by surface self-diffusion, a grain boundary groove width has been given by the following Mullins' equation $^{(8)}$ :

$$
\begin{aligned}
& w=4.6(B t)^{1 / 4}, \\
& B=D_{s} \gamma_{s} \Omega^{2} N / k T,
\end{aligned}
$$

where $D_{s}$ is a surface self-diffusion coefficient, $\gamma_{s}$ is the surface tension, $\Omega$ is the atomic volume and $N$ is the atomic density at a surface.

Buttner et al. has reported $\gamma_{s}$ of silver at $1205 \mathrm{~K}$ as follows ${ }^{(9)}$ :

$$
\begin{aligned}
& P_{\mathrm{O}_{2}}<10 \mathrm{~Pa}, \gamma_{s}=1.14 \mathrm{~N} / \mathrm{m}, \\
& 10 \mathrm{~Pa}< P_{\mathrm{O}_{2}}<10^{5} \mathrm{~Pa}, \gamma_{s} \\
&=\left\{288-188 \log \left(P_{\mathrm{O}_{2}} / 1.013 \times 10^{5}\right)\right\} \\
& \times 10^{-3} \mathrm{~N} / \mathrm{m},
\end{aligned}
$$

In this experiment, these values were used. 

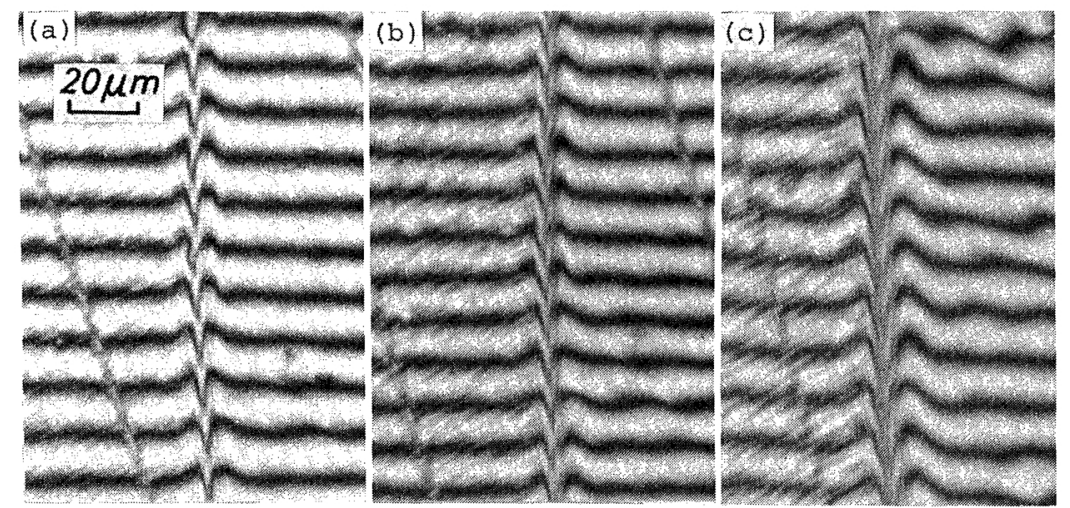

Photo. 1 The grain boundary grooves at $1023 \mathrm{~K}$ in an argon atmosphere with $P_{\mathbf{O}_{2}}=10^{-11} \mathrm{~Pa}$.

(a) $18 \mathrm{ks}$, (b) $36 \mathrm{ks}$, (c) $180 \mathrm{ks}$

\section{Results}

\section{The measurements of the surface} self-diffusion coefficient at $P_{0_{2}}=10^{-11} \mathrm{~Pa}$

The surface self-diffusion coefficients of silver has been already obtained in several different atmospheres. In the present experiment the surface self-diffusion coefficient was at first obtained in the very low oxygen potential which was considered to be free from the adsorption of oxygen $\left(P_{\mathrm{O}_{2}}=10^{-11} \mathrm{~Pa}\right)$ and compared with other reported values.

Photograph 1 shows the growth of the grain boundary grooves at $1023 \mathrm{~K}$. As the time passed, the grooves were widened and deepened. Figure 1 shows the time dependence of the growth of the grain boundary groove widths. At each temperature the logarithm of the grain boundary groove widths has a linear relation with that of annealing time and its slope was $0.25 \pm 0.01$. This showed from eq. (1) that the grain boundary grooves were formed by surface self-diffusion. The logarithms of the surface self-diffusion coefficients were plotted against $1 / T$. This is shown by the solid line in Fig. 2 and could be expressed by eq. (2).

$$
\begin{array}{r}
D_{s}=0.249 \exp (-166 \mathrm{~kJ} / \mathrm{mol} \cdot R T) \mathrm{m}^{2} / \mathrm{s} \\
(973 \sim 1073 \mathrm{~K})
\end{array}
$$

The surface self-diffusion coefficients in various atmospheres measured by other researchers and also the values calculated from Gjostein's experimental formula for $f c c$ metals $^{(10)}$ are shown in Fig. 2. The diffusion coefficient in

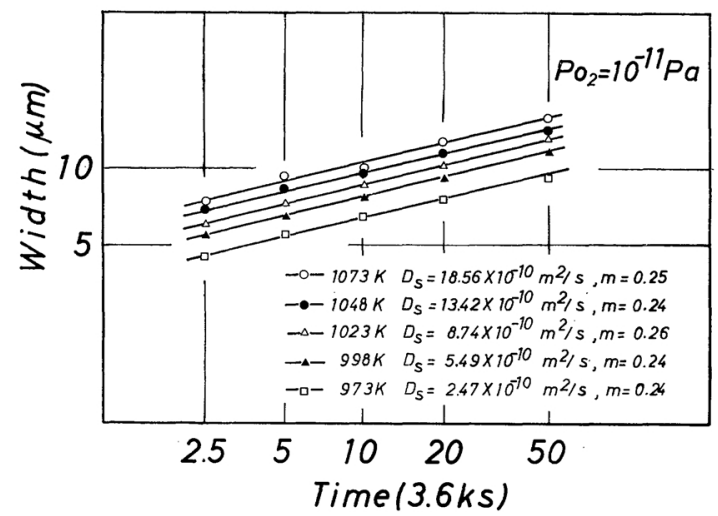

Fig. $1 \log w$ vs $\log t$ at $P_{\mathrm{O}_{2}}=10^{-11} \mathrm{~Pa}$.

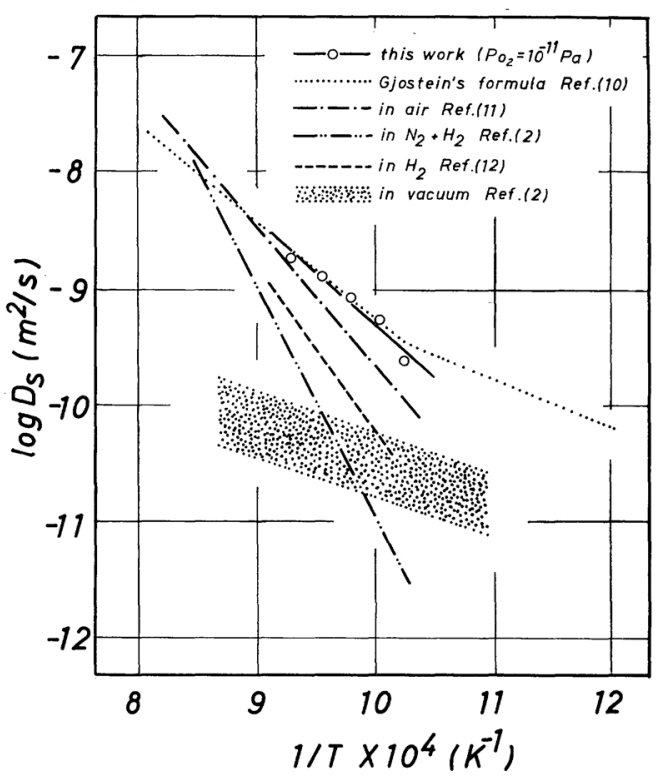

Fig. 2 The surface self-diffusion coefficient for silver in various atmospheres. 
this experiment coincides well with Gjostein's formula. As for the previously reported values in Fig. 2, the surface self-diffusion coefficient of silver in air is found to be larger than those in other atmospheres. It has been reported that in a high pressure oxygen atmosphere the surface self-diffusion coefficient of silver increases as the oxygen pressure increased ${ }^{(2)}$.

\section{The measurements of surface} self-diffusion coefficient at various oxygen potentials between $10^{-11} \mathrm{~Pa}$ and $10^{5} \mathrm{~Pa}$ at $1023 \mathrm{~K}$

It is deduced from Fig. 2 that the surface self-diffusion coefficient is strongly affected by oxygen potentials. The grain boundary groove widths were measured at various oxygen potentials between $10^{-11}$ and $10^{5} \mathrm{~Pa}$ at $1023 \mathrm{~K}$. Before measurements, it was necessary to anneal the sample for $50 \mathrm{~h}$ at the same oxygen potential as that of diffusion anneal in order to equilibrate the oxygen potential between the sample and the atmosphere ${ }^{(1)}$. The measured grain boundary groove widths and the annealing time were both plotted on a logarithmic scale and the slope at each oxygen potential was $0.25 \pm 0.01$. Therefore the grain boundary grooves were formed by surface self-diffusion at these oxygen potentials. The surface selfdiffusion coefficients were calculated and plotted against oxygen potentials. The result is shown in Fig. 3. Figure 3 shows that as the oxygen potential increases from $P_{\mathrm{O}_{2}}=10^{-11}$ $\mathrm{Pa}$, the surface self-diffusion coefficient de-

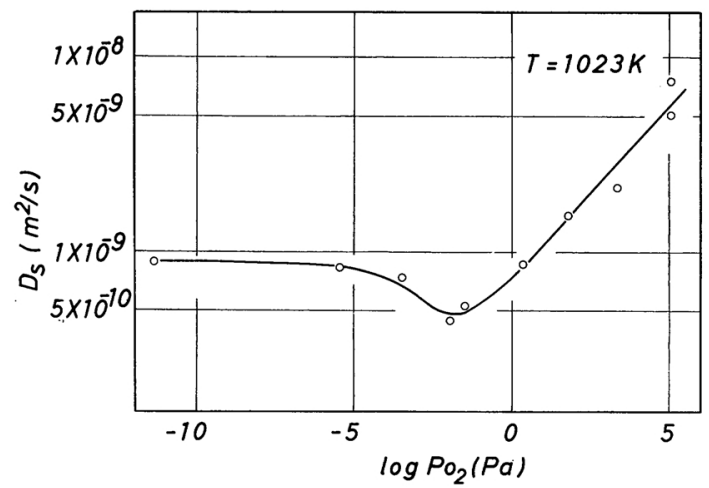

Fig. 3 The relationship between the surface selfdiffusion coefficient and the oxygen potential at $1023 \mathrm{~K}$.

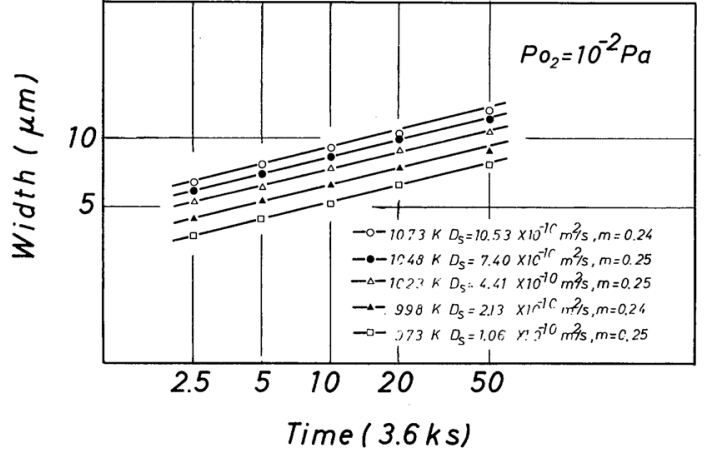

Fig. $4 \log w$ vs $\log t$ at $P_{\mathrm{O}_{2}}=10^{-2} \mathrm{~Pa}$.

creases and becomes minimum at $P_{\mathrm{O}_{2}}=10^{-2}$ $\mathrm{Pa}$ and as the oxygen potential further increases above $P_{\mathrm{O}_{2}}=10^{-2} \mathrm{~Pa}$, the surface self-diffusion coefficient increases again. This tendency must be caused by the change of the states of oxygen adsorption on the silver surface. Therefore the temperature dependence of surface self-diffusion coefficient was measured at $P_{\mathrm{O}_{2}}=10^{-2} \mathrm{~Pa}$ and at $P_{\mathrm{O}_{2}}=10^{5} \mathrm{~Pa}$.

\section{The measurements of surface} self-diffusion coefficient at $P_{\mathrm{O}_{2}}=10^{5} \mathrm{~Pa}$

Keeping the oxygen potential at $10^{-2} \mathrm{~Pa}$, grain boundary groove widths were measured varying the annealing temperature between 973 and $1073 \mathrm{~K}$. Before measurements, the sample was preannealed for $50 \mathrm{~h}$ at the same tempeature of diffusion anneal in order to equilibrate the oxygen potential between the sample and the atmosphere. The time dependence of the grain boundary groove widths is shown in Fig. 4. The slope at each temperature is $0.25 \pm 0.01$. Therefore, the grain boundary grooves were formed by surface self-diffusion.

\section{The measurements of surface self-diffusion coefficient at $P_{\mathrm{O}_{2}}=10^{5} \mathrm{~Pa}$}

Grain boundary groove widths were measured in an oxygen atmosphere $\left(P_{\mathrm{O}_{2}}=10^{5} \mathrm{~Pa}\right)$ at temperatures between 973 and $1073 \mathrm{~K}$. Before measurements, the sample was also annealed for $50 \mathrm{~h}$ at the diffusion anneal temperature. The growth of the grain boundary grooves are shown in Photo. 2. Comparison between Photo. 1 and Photo. 2 shows that facetings occur at $P_{\mathrm{O}_{2}}=10^{5} \mathrm{~Pa}$. The time 

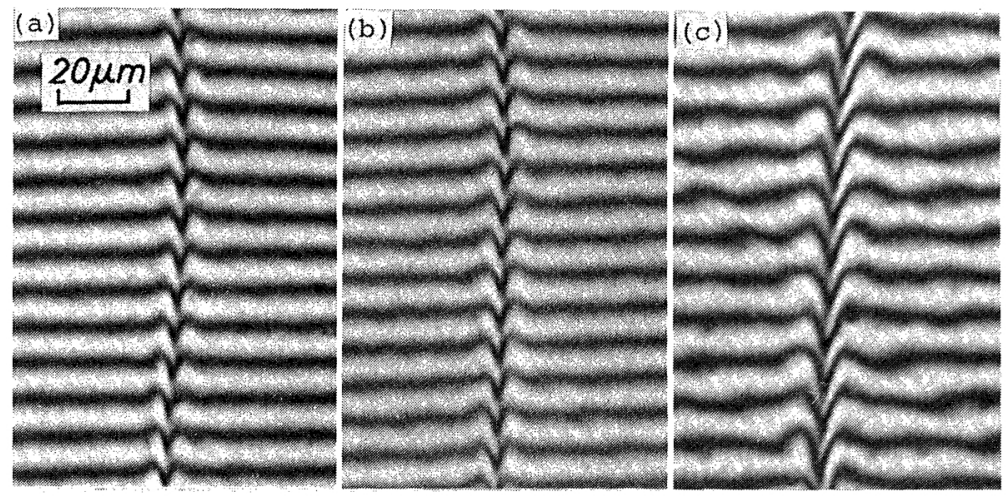

Photo. 2 The grain boundary grooves at $1023 \mathrm{~K}$ in an oxygen atmosphere $\left(P_{\mathrm{O}_{2}}=10^{5} \mathrm{~Pa}\right)$. (a) $18 \mathrm{ks}$, (b) $36 \mathrm{ks}$, (c) $180 \mathrm{ks}$

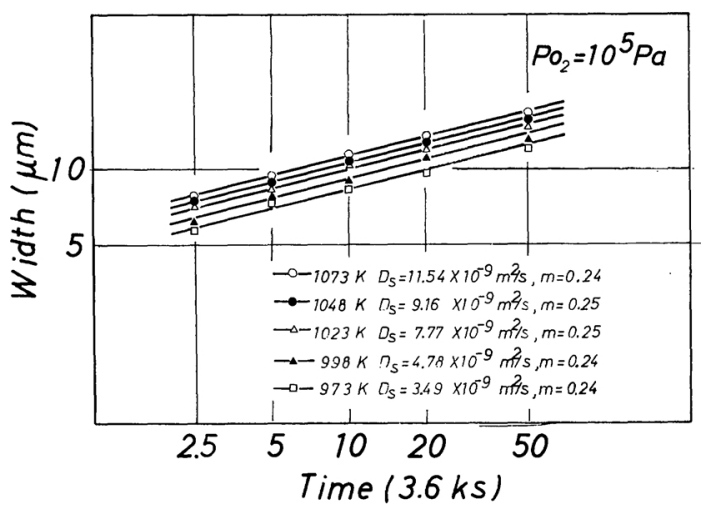

Fig. $5 \log w$ vs $\log t$ at $P_{\mathrm{O}_{2}}=10^{5} \mathrm{~Pa}$.

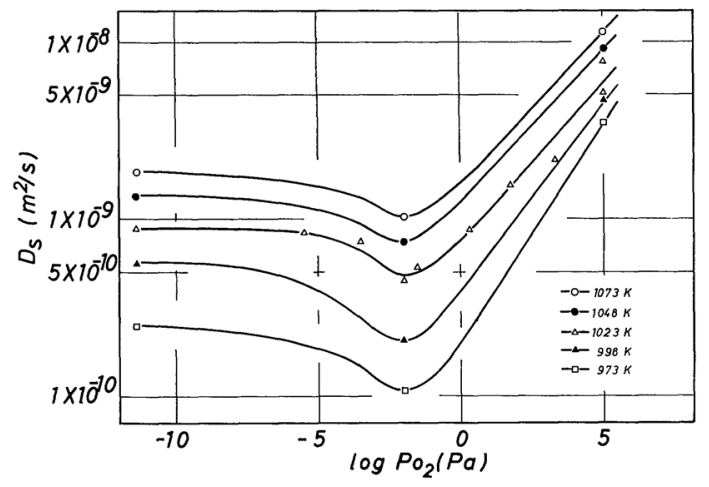

Fig. 6 The relationship between the surface selfdiffusion coefficient and the oxygen potential.

dependence of grain boundary groove widths is shown in Fig. 5. The slope at each temperature is $0.25 \pm 0.01$. Therefore, the development of grain boundary grooves is surface selfdiffusion dependent.

\section{Discussion}

The surface self-diffusion coefficient at each oxygen potential and temperature is measured, and plotted against the oxygen potential. The results are shown in Fig. 6. Figure 6 shows that as the oxygen potential increases from $P_{\mathrm{O}_{2}}=10^{-11} \mathrm{~Pa}$, the surface self-diffusion coefficient decreases and becomes minimum at $P_{\mathrm{O}_{2}}=10^{-2} \mathrm{~Pa}$ and as the oxygen potential further increases above $P_{\mathrm{O}_{2}}=10^{-2} \mathrm{~Pa}$, it increases. The logarithms of the surface selfdiffusion coefficients are plotted against $1 / T$. The results are shown in Fig. 7. Figure 7 shows that at $P_{\mathrm{O}_{2}}=10^{-11} \mathrm{~Pa}$ and at $P_{\mathrm{O}_{2}}=10^{5} \mathrm{~Pa}$, the lines are straight. The surface self-diffusion coefficient at $P_{\mathrm{O}_{2}}=10^{5} \mathrm{~Pa}$ was larger than that at $P_{\mathrm{O}_{2}}=10^{-11} \mathrm{~Pa}$. The surface self-diffusion coefficient at $P_{\mathrm{O}_{2}}=10^{-11} \mathrm{~Pa}$ was already given by eq. (2). At $P_{\mathrm{O}_{2}}=10^{5} \mathrm{~Pa}$, it was expressed by the following equation:

$$
\begin{array}{r}
D_{s}=1.71 \times 10^{-3} \exp (-106 \mathrm{~kJ} / \mathrm{mol} . R T) \mathrm{m}^{2} / \mathrm{s} \\
(973 \sim 1073 \mathrm{~K}) .
\end{array}
$$

The activation energy at $P_{\mathrm{O}_{2}}=10^{5} \mathrm{~Pa}$ is smaller than $166 \mathrm{~kJ} / \mathrm{mol}$ at $P_{\mathrm{O}_{2}}=10^{-11} \mathrm{~Pa}$. At $P_{\mathrm{O}_{2}}=$ $10^{-2} \mathrm{~Pa}$, as shown in Fig. 7, the surface selfdiffusion coefficient was smaller than either at $P_{\mathrm{O}_{2}}=10^{-11} \mathrm{~Pa}$ or at $P_{\mathrm{O}_{2}}=10^{5} \mathrm{~Pa}$, and its temperature dependence could not be expressed by Arrhenius' equation. To interprete the above results, the change of the surface structure of silver with oxygen potential has been investigated as follows. 


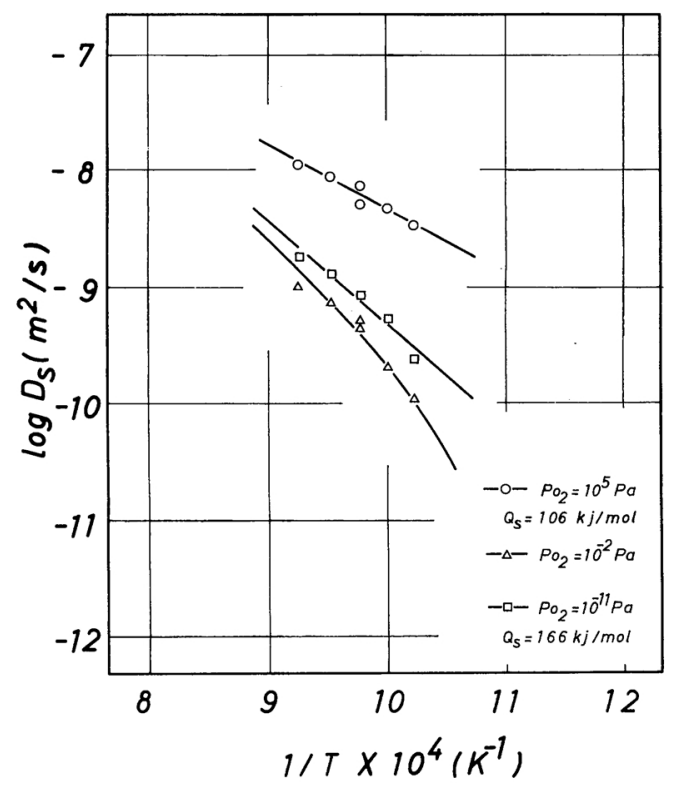

Fig. 7 The temperature dependence of the surface self-diffusion coefficient.

It is convenient to use the TLK (TerraceLedge-Kink) model in order to simply explain the migration of an atom on a metal surface. A surface self-diffusion coefficient can be expressed by the migration of ad-atoms and surface vacancies on a metal surface. Here, only the migrations of ad-atoms will be considered since the migration of surface vacancies are expressed in a way similar to that of ad-atoms. The following equilibrium is assumed to be existing on a metal surface so that a ledge site and an ad-atom may be in an equilibrium:

Ledge site $\rightleftarrows$ kink site $\rightleftarrows$ ledge ad-atom $\rightleftarrows$ ad-atom.

Although the relation between a terrace site and a ledge site is determined by the geometrical relation of a crystal surface, here a terrace site and an ad-atom are supposed to be in a thermal equilibrium for simplicity. Then, the number of ad-atoms per unit area, $n_{a}$, is given by the following equation:

$$
n_{a}=N \exp \left(-\Delta G_{f}^{a} / k T\right),
$$

where $N$ is the number of terrace sites per unit area and $\Delta G_{f}^{a}$ is the free energy of formation of an ad-atom from a terrace site. The surface self-diffusion coefficient $D_{s}$ is expressed by the following equation ${ }^{(10)}$ :

$$
D_{s}=\left(n_{a} / N\right) D_{a} .
$$

Here $D_{a}$ is the diffusion coefficient of an adatom in the form

$$
D_{a}=\alpha l^{2} v \exp \left(-\Delta G_{m}^{a} / k T\right),
$$

where $\alpha$ is a dimensionality constant, $l$ is an average jump distance, $v$ is a vibrational frequency of an ad-atom and $\Delta G_{m}^{a}$ is a free energy of the migration of an ad-atom. By putting eq. (6) and eq. (4) into eq. (5), we obtain

$$
\begin{aligned}
D_{s} & =\alpha^{2} v \exp \left[-\left(\Delta G_{f}^{a}+\Delta G_{m}^{a}\right) / k T\right] \\
& =D_{0} \exp \left(-\Delta H_{s} / k T\right),
\end{aligned}
$$

where

$$
\begin{aligned}
& D_{0}=\alpha l^{2} v \exp \left[\left(\Delta S_{f}^{a}+\Delta S_{m}^{a}\right) / k\right], \\
& \Delta H_{s}=\Delta H_{f}^{a}+\Delta H_{m}^{a} .
\end{aligned}
$$

In order to characterize the surface structure of silver at each oxygen potential, the following assumptions are introduced.

(1) As for the orientation of silver surface, (111) surface is considered. Only ad-atoms move on this surface, and the equilibrium between terrace site and an ad-atom is established.

(2) Morse potential is used for calculating the binding energy of metal atoms. Morse potential has been given by the following equation:

$$
\begin{aligned}
\varphi\left(r_{i j}\right)= & D\left[\exp \left\{-2 \alpha\left(r_{i j}-r_{0}\right)\right\}\right. \\
& \left.-2 \exp \left\{-\alpha\left(r_{i j}-r_{0}\right)\right\}\right]
\end{aligned}
$$

The values of $D, \alpha$ and $r_{0}$ for silver have been given by Gilifalco et al. ${ }^{(13)}$

(3) An adsorbed oxygen is ionized completely. If the amount of adsorbed oxygen is small and an adsorbed oxygen is isolated, neighboring silver atoms are ionized. As the amount of adsorbed oxygen increases and adsorbed oxygens are arranged in order, all the surface silver atoms are ionized equally so as to cancel the charge of the adsorbed oxygens. In this case, a 2-dimensional ionic crystal is formed on the surface. Coulomb's force acts on the ion-ion interaction. However, if the ionic valence of $+1 / 6$ is allotted to one 


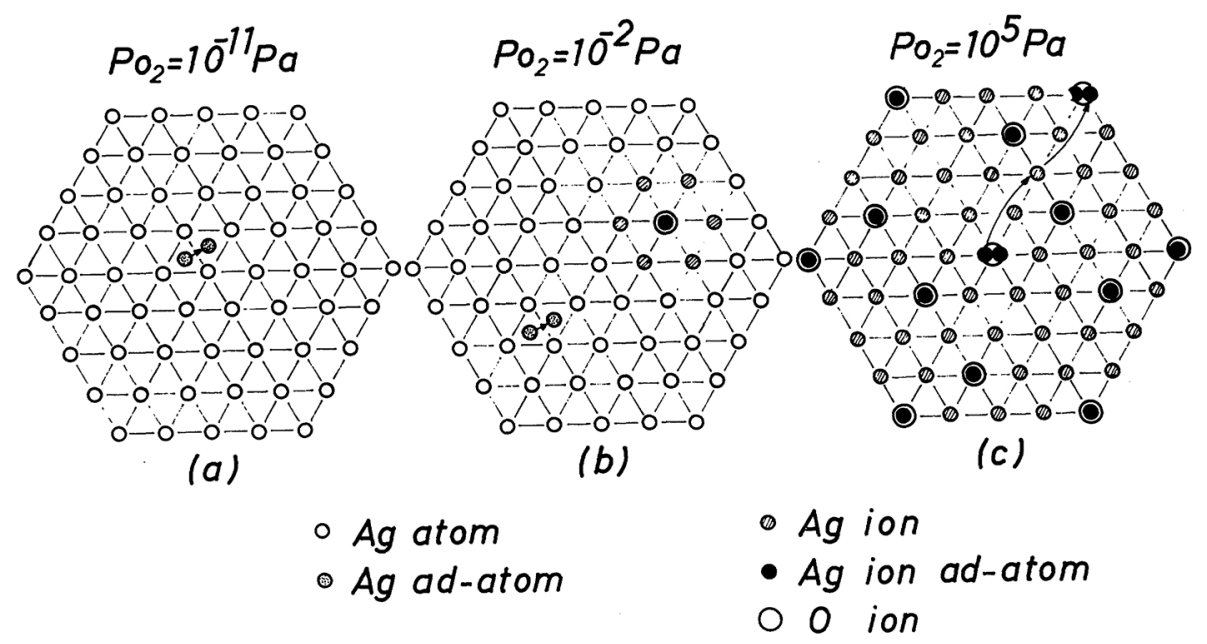

Fig. 8 The model for the surface structure of silver.

silver atom around an adsorbed oxygen ion, Morse potential force is assumed to act on the rest of the atom, 5/6.

(4) Adsorbed oxygen ions take $(4 \times 4)$ structure on the silver (111) surface ${ }^{(14)}$.

(5) The change of the vibrational frequency of an ad-atom is neglected for simplicity.

(6) The relaxation of the surface is not considered. Namely, the atomic distance of surface atoms is the same as that of bulk and does not change by the adsorption of oxygen.

Based on these assumptions, the model of the surface structures of silver at various oxygen potentials is proposed as follows:

(a) $P_{\mathrm{O}_{2}}=10^{-11} \mathrm{~Pa}$.

There is no adsorption of oxygen on the surface. The bonding of silver atoms at terrace sites is metallic. This model is shown in Fig. 8(a). An ad-atom diffuses on the terrace as shown in Fig. 8(a). The binding energy is calculated by using Morse potential. The potential of an ad-atom at the stable point on the terrace is $-95 \mathrm{~kJ} / \mathrm{mol}$ and that at the saddle point of the diffusion path is -64 $\mathrm{kJ} / \mathrm{mol}$. The potential at a terrace site is -286 $\mathrm{kJ} / \mathrm{mol}$. The calculated energy diagram is shown in Fig. 9(a). Then the enthalpy of formation of an ad-atom, $\Delta H_{f}^{a}$, is $191 \mathrm{~kJ} / \mathrm{mol}$ and that of migration of an ad-atom, $\Delta H_{m}^{a}$, is $31 \mathrm{~kJ} / \mathrm{mol}$. Therefore, the activation energy of diffusion, $\Delta H_{s}=\Delta H_{f}^{a}+\Delta H_{m}^{a}$, is $222 \mathrm{~kJ} / \mathrm{mol}$. The jump distance, $l$, is $1.67 \times 10^{-10} \mathrm{~m}$. (b) $P_{\mathrm{O}_{2}}=10^{-2} \mathrm{~Pa}$.

It is assumed that the amount of adsorbed oxygens is small and oxygen ions are isolated on the surface. This model is shown in Fig. 8(b). On the terrace sites where there is no adsorbed oxygen, an ad-atom diffuses in the same way as on the clean surface at $P_{\mathrm{O}_{2}}=$ $10^{-11} \mathrm{~Pa}$. The silver atoms at the neighboring terrace sites of an adsorbed oxygen ion are partially ionized. Then the potential of the terrace site on which an oxygen ion is adsorbed is $-495 \mathrm{~kJ} / \mathrm{mol}$. Therefore, the silver atom becomes very stable. If an ad-atom climbs on the adsorbed oxygen ion as shown in Fig. $8(\mathrm{~b})$, its potential is $-346 \mathrm{~kJ} / \mathrm{mol}$ and it is strongly bound to oxygen ion. Therefore, the ad-atom which forms a pair with an adsorbed oxygen ion cannot move, so it does not contribute to the diffusion. The energy diagram in this case is shown in Fig. 9(b). Here, $n_{a m}$ is the number of ad-atoms which can contribute to the diffusion. The neighboring silver atoms around an adsorbed oxygen ion are stabilized because of Coulomb's interaction between the partially ionized silver ion and the adsorbed oxygen ion. Therefore, ad-atoms cannot be formed from these terrace sites. The number of terrace sites per unit area from which adatoms can be produced, $N_{f}$, is expressed as follows:

$$
N_{f}=N-\beta N_{0}
$$



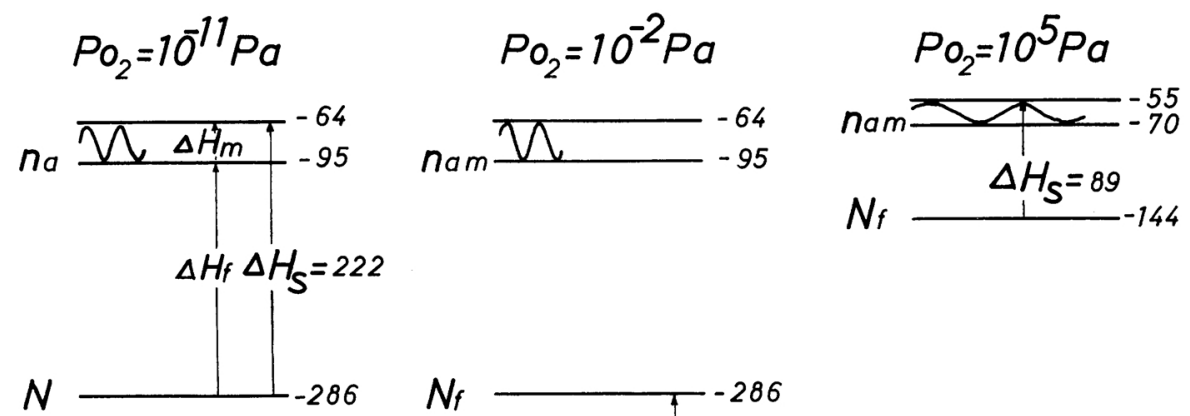

(a) (b) (c)

Fig. 9 The calculated energy diagram for the surface of silver (unit: $\mathrm{kJ} / \mathrm{mol}$ ).

where $N_{0}$ is the number of terrace sites per unit area where oxygen ions are adsorbed, and $\beta N_{0}$ is that of the terrace sites which are stabilized by oxygen ions $(\beta=7)$. Now the following equilibrium is supposed:

$$
N_{f}+1 / 2 \mathrm{O}_{2} \rightleftarrows N_{0} \text {. }
$$

Hence, we obtain

$$
K=N_{0} / N_{f}\left(P_{\mathrm{O}_{2}}\right)^{1 / 2}=\exp \left(-\Delta G_{f}^{\prime} / k T\right)
$$

where $\Delta G_{f}^{\prime}$ is the energy difference between the terrace sites with and without adsorption of the oxygen ion. Its calculated enthalpy is shown in Fig. 9(b). From eqs. (9) and (10),

$$
N_{f}=N\left\{1+\beta P_{\mathrm{O}_{2}}^{1 / 2} \exp \left(-\Delta G_{f}^{\prime} / k T\right)\right\}^{-1} \text {. }
$$

From eqs. (4) and (11),

$$
\begin{aligned}
n_{a m}= & N\left\{1+\beta P_{\mathrm{O}_{2}}^{1 / 2} \exp \left(-\Delta G_{f}^{\prime} / k T\right)\right\}^{-1} \\
& \times \exp \left(-\Delta G_{f}^{a} / k T\right) .
\end{aligned}
$$

From eqs. (5), (6) and (12),

$$
\begin{aligned}
D_{s}= & D_{0} \exp \left(-\Delta H_{s} / k T\right) \\
& \times\left\{1+\beta P_{\mathrm{O}_{2}}^{1 / 2} \exp \left(-\Delta G_{f}^{\prime} / k T\right)\right\}^{-1} .
\end{aligned}
$$

Because $\left\{1+\beta P_{\mathrm{O}_{2}}^{1 / 2} \exp \left(-\Delta G_{f}^{\prime} / k T\right)\right\}^{-1}<1, D_{s}$ at $10^{-2} \mathrm{~Pa}$ is smaller than that on clean surface. Namely, the diffusion coefficient decreases with the increase of the amount of adsorbed oxygen, since the number of ad-atoms which contribute to the diffusion decreases [eq. (12)].
Equation (13) shows as the oxygen potential increases keeping an annealing temperature constant, the surface self-diffusion coefficient decreases. This tendency coincides with the experimental result shown in Fig. 6 that the surface self-diffusion coefficient decreased as the oxygen potential increased from $10^{-11} \mathrm{~Pa}$ to $10^{-2} \mathrm{~Pa}$. As the annealing temperature decreases at a constant oxygen potential, the term $\exp \left(-\Delta G_{f}^{\prime} / k T\right)$ increases. Therefore, $\log D_{s}$ is not proportional to $1 / T$ and the shape of line in Arrehenius plot becomes convex as shown by eq. (13). This tendency also coincides with the experimental results shown in Fig. 7. It has been reported that the logarithm of the surface self-diffusion coefficient of iron had not been proportional to $1 / T$ in a wet hydrogen atmosphere ${ }^{(1)}$. This fact is also explained by eq. (13).

(c) $P_{\mathrm{O}_{2}}=10^{5} \mathrm{~Pa}$.

The saturated amount of oxygen ions is adsorbed. The oxygen ions which have been isolated at $P_{\mathrm{O}_{2}}=10^{-2} \mathrm{~Pa}$ are now arranged in order and $(4 \times 4)$ structure is formed. This model is shown in Fig. 8(c). The 2-dimensional ionic crystal is formed on the surface. The adsorbed oxygen atoms are totally ionized, and silver atoms are partially ionized so as to keep the electro-neutrality of the surface. Based on these assumptions, the potential at 
each surface site is calculated. From this calculation, it is deduced that an ad-atom of silver ion migrates through the path indicated by the arrows in Fig. 8(c). The potential on an oxygen ion and that at saddle point are $-70 \mathrm{~kJ} / \mathrm{mol}$ and $-55 \mathrm{~kJ} / \mathrm{mol}$ respectively. The binding energy of a terrace site is $-144 \mathrm{~kJ} / \mathrm{mol}$. This energy diagram is shown in Fig. 9(c). The enthalpy of formation, $\Delta H_{f}^{a}$, and that of motion of an ad-atom, $\Delta H_{m}^{a}$, are $74 \mathrm{~kJ} / \mathrm{mol}$ and $15 \mathrm{~kJ} / \mathrm{mol}$ respectively. Therefore, the activation energy of diffusion is $89 \mathrm{~kJ} / \mathrm{mol}$, which is smaller than the calculated value at $P_{\mathrm{O}_{2}}=10^{-11} \mathrm{~Pa}$. This suggests that the potential barrier for the diffusion decreases by the overlapping of long range Coulomb's force when the 2-dimensional ionic crystal is formed. The tendency of this calculation coincides with the experimental results that the activation energy at $P_{\mathrm{O}_{2}}=10^{5} \mathrm{~Pa}, 106 \mathrm{~kJ} / \mathrm{mol}$, was smaller than that at $P_{\mathrm{O}_{2}}=10^{-11} \mathrm{~Pa}, 166 \mathrm{~kJ} / \mathrm{mol}$. The jump distance is $1.156 \times 10^{-9} \mathrm{~m}$, which is larger than that on the clean surface. Therefore, the surface self-diffusion coefficient should be large when the amount of adsorption reaches the saturated value.

As shown in Fig. 6, however, the surface self-diffusion coefficient increased continuously as the oxygen potential increased from $10^{-2}$ to $10^{5} \mathrm{~Pa}$. This is explained by the assumption that the patches where the 2-dimensional ionic crystal is formed exist on the surface where adsorbed oxygen ions are isolated. The thermodynamical explanation of the 2-phase coexistence on the surface at an appropriate temperature has been already given by Blakely et al. ${ }^{(15)}$ The surface self-diffusion coefficient is large at the patches where the adsorption is saturated and it is small at these where adsorbed oxygen ions are isolated. As the oxygen potential increases, the patch where the adsorption is saturated grows. As a result, the surface selfdiffusion coefficient increases.

It has been reported that the surface selfdiffusion coefficient of iron had decreased and become minimum then increased as the oxygen potential increased ${ }^{(16)}$. This result coincides with the experiments of silver, and is also explained by the same mechanism as silver. Namely, with the increase in oxygen potential, the ionic character of the surface is enhanced and the surface self-diffusion coefficient is increased.

\section{Conclusion}

The effect of the oxygen potential on the surface self-diffusion coefficient of silver has been obtained by the grain boundary grooving method. The results were as follows:

(1) The surface self-diffusion coefficient at $P_{\mathrm{O}_{2}}=10^{-11} \mathrm{~Pa}$ could be expressed by the following equation:

$$
\begin{aligned}
D_{s}= & 0.249 \exp (-166 \mathrm{~kJ} / \mathrm{mol} \cdot R T) \mathrm{m}^{2} / \mathrm{s} . \\
& (973 \sim 1073 \mathrm{~K})
\end{aligned}
$$

(2) The surface self-diffusion coefficient at $P_{\mathrm{O}_{2}}=10^{5} \mathrm{~Pa}$ could be expressed by the following equation:

$$
\begin{aligned}
D_{s}= & 1.71 \times 10^{-3} \exp (-106 \mathrm{~kJ} / \mathrm{mol} \cdot R T) \mathrm{m}^{2} / \mathrm{s} . \\
& (973 \sim 1073 \mathrm{~K})
\end{aligned}
$$

(3) The surface self-diffusion coefficient at $P_{\mathrm{O}_{2}}=10^{-2} \mathrm{~Pa}$ was smaller either at $P_{\mathrm{O}_{2}}=$ $10^{-11} \mathrm{~Pa}$ or at $P_{\mathrm{O}_{2}}=10^{5} \mathrm{~Pa}$ and it could be expressed by the following equation and not by Arrhenius' equation:

$$
\begin{aligned}
D_{s}= & D_{0} \exp \left(-\Delta H_{s} / k T\right) \\
& \times\left\{1+\beta P_{\mathrm{O}_{2}}^{1 / 2} \exp \left(-\Delta G_{f}^{\prime} / k T\right)\right\}^{-1} .
\end{aligned}
$$

(4) The surface self-diffusion coefficient of silver once decreased and became minimum, then increased, as the oxygen potential increased. This was explained by the following model. When oxygen potential is small, adsorbed oxygen ions are isolated, and only neighboring silver atoms are ionized and stabilized. Then the number of terrace sites, from which mobile ad-atoms are produced decreases, and thus the diffusivity decreases. As the oxygen potential increases further, adsorbed oxygen ions are arranged in order, and the 2-dimensional ionic crystal is formed. Then the jump distance becomes longer and the activation energy for the diffusion becomes smaller by an overlapping of long range Coulomb's force. As a result, the surface selfdiffusion coefficient increases.

(5) The surface self-diffusion coefficient of metals is affected remarkably by the oxygen potential of an atmosphere. This is explained by the change of ionic character of the surface 
with the variation of the oxygen potential, as shown in the present paper. This model is considered to be valid for metals in general.

\section{REFERENCES}

(1) K. Nii and K. Yoshihara: J. Japan Inst. Metals, 40 (1976), 26.

(2) G. E. Rhead: Acta Met., 13 (1965), 223.

(3) G. E. Rhead: Surface Sci., 15 (1969), 353.

(4) H. P. Bonzel and N. A. Gjostein: ibid., 22 (1970), 216.

(5) H. P. Bonzel: Structure and Properties of Metal Surfaces, Honda Memorial Series on Material Science No. 1, Ed. by S. Shimodaira, M. Maeda, G. Okamoto, M. Onchi and Y. Tamai, Maruzen, Tokyo, (1973), p. 248.

(6) M. McLean and J. P. Hirth: Surface Sci., 12 (1968), 177.

(7) F. Cabané-Brouty and B. Ruzé: Métaux, Corrosion Industries, (1964), 343.
(8) W. W. Mullins: J. Appl. Phys., 28 (1957), 333.

(9) F. H. Buttner, E. R. Funk and H. Udin: J. Phys. Chem., 56 (1952), 657.

(10) N. A. Gjostein: Surfaces and Interfaces I, Proceedings of the 13th Sagamore Army Materials Research Conference, Ed. by J. J. Burke, N. L. Reed and V. Weiss, Syracuse University Press, Syracuse, (1967), p. 271.

(11) G. E. Rhead: Acta Met., 11 (1963), 1035.

(12) R. R. Hough: Scripta Met., 4 (1970), 559.

(13) L. A. Girifalco and V. G. Weizer: Phys. Rev., 114 (1959), 687.

(14) G. Rovida, F. Pratesi, M. Magrietta and E. Ferroni: Surface Sci., 43 (1974), 230.

(15) J. M. Blakely and J. C. Shelton: Surface Physics of Materials, Ed. by J. M. Blakely, Academic Press, New York, San Francisco, London, (1975), p. 189.

(16) K. Yoshihara and K. Nii: Collected Abstracts of the 1976 Spring Meeting of Japan Inst. Metals, p. 98. 